\title{
Clinical effectiveness of electrical fire needling for shoulder pain of amyotrophic lateral sclerosis: a pilot study
}

\begin{abstract}
Amyotrophic lateral sclerosis (ALS) is a progressive and incurable disease that causes degeneration of the motor neurons of the brain stem and the spinal cord. About half of ALS patients reported pain and joint contracture. Especially, the pain and contracture in shoulder joint are the main causes of decreasing quality of patient's life.

Electrical fire needling is a therapeutic measure of applying the stimulation of both acupuncture and heating. And it has effects of relieving pain and joint contracture. ALS patients with shoulder pain who visited the department of Acupuncture \& Moxibustion in Wonkwang Gwangju Medical Center had taken electrical fire needling therapy for 8times. We evaluated the relief of their shoulder pain and disability using the Visual Analog Scale (VAS), and Passive Range of Motion (PROM). The pain evaluated by VAS was gradually decreased, and there was a significant difference between study group and control group. The mobility calculated by ROM was generally increased, but there was no significant difference between study group and control group generally.

The above results show that electrical fire needling can be used as an effective treatment for reducing shoulder pain. Further studies on this subject are needed to yield more concrete evidence.
\end{abstract}

Volume 4 Issue 2 - 2016

Sungchul Kim, I,2 Eunhye Cha, I,2 Seongjin Lee, I, 2 June Hyun Kim,3 Dong Woung Kim,4 Jong Deok Lee5

'Department of Acupuncture \& Moxibustion Medicine, Wonkwang University, Korea

${ }^{2}$ Nervous \& Muscular System Disease Clinical Research Center of Wonkwang University, Korea

${ }^{3}$ Department of Acupuncture \& Moxibustion Medicine,

Wonkwang University, Korea

${ }^{4}$ Department of Internal Medicine, Wonkwang University, Korea 5Department of Radiology, Wonkwang University, Korea

Correspondence: Sungchul Kim, Wonkwang Gwangju Oriental Medical Hospital, 543-8 Juweol I-dong, Nam-gu, Gwangju, 503310, Korea, Tel +82-62-670-644I, Email kscndl@hanmail.net

Received: July 27, 2016 | Published: August 10, 2016

Keywords: amyotrophic lateral sclerosis(als), shoulder pain, electrical fire needling

\section{Introduction}

Amyotrophic lateral sclerosis (ALS) is a neurological disease of unknown origin characterized by selective degeneration and death of upper and lower motor neurons. It almost invariably progresses to paralysis and death over a 1-5year time course. ${ }^{1}$ At present, there is no therapy that can reverse loss of functions. ${ }^{2}$ In oriental medicine, Wei symptom (瘘證) is similar to as ALS. ${ }^{3}$ Wei symptom means limb paralysis due to muscle atony that cannot make a muscle contract. It begins as mild degree from extremities, but in some cases ends to quadriplegia or death. ${ }^{4}$

Pain is a frequent symptom in ALS, especially in the later stages of disease $^{5}$ and influences quality of life, increasing suffering and hopelessness. ${ }^{6}$ Previous studies report that about $50 \%{ }^{7}$ to more than $70 \%^{8}$ of ALS patients complain pain. It is believed that an indirect symptom of pathology occurs, as a result of primary symptoms of muscular weakening. Musculoskeletal pain is often related to atrophy and altered tone around joints, but also muscle contracture and joint stiffness can cause patients sever pain. ${ }^{9}$ With the decreasing of mobility, pain becomes more severe, so it may result in frozen joints or inability when the patients try to shift their positions. ${ }^{10}$

Especially, the pain and contracture in shoulder joint are the main causes of decreasing quality of patient's life. ${ }^{3}$ ALS patient's upper and lower limbs are getting weaker so the muscle and joint contracture may be more rapidly progressive and difficult to recover.

From past to present, a lot of treatment methods have been developed to treat shoulder pain. ${ }^{11}$ Among them, fire needling is a treatment method that an illness is cured through an instantaneous injection of a red-hot needle ${ }^{12}$ so from ancient times, a fire needling is used to treat many kinds of diseases such as an arthralgia syndrome and a musculoskeletal disease. ${ }^{13}$
The symptom of the shoulder pain that amylotrophic lateral sclerosis (ALS) patients have commonly suffered has many cases of muscle contraction as mentioned earlier, so is considered to fall under the category of arthralgia syndrome. On this account, electrical fire needling device has been used to apply the treatment of fire needling to shoulder pain for ALS patients. Several studies on effectiveness fire needling were conducted. Nevertheless, the study had not been applied to patients with shoulder pain of amyotrophic lateral sclerosis yet.

Therefore, this study was conducted to study the effect of electrical fire needling treatment for shoulder pain of amyotrophic lateral sclerosis (ALS).

\section{Materials and methods}

\section{Subjects}

This study was conducted at the Wonkwang University ALS clinic from February 2014 to October 2015. Eighteen eligible ALS patients were selected from the patients who agreed on the clinical trial.

\section{A. Inclusion criteria}

a. Patients who satisfied the EL Escorial criteria and who were diagnosed with ALS by EMG in general hospital

b. Patients who signed consent

c. Patients who complain shoulder pain with more than VAS 5

\section{B. Exclusion criteria}

a. Patients who were not able to give basic information due to the severe bulbar palsy

b. Patients who were addicted to drugs such as alcohol, nicotine, or caffeine 
c. Patients who take analgesic medicine or another medical treatment

d. Patients who have cervical origin shoulder pain in physical exam

e. Patients who use pacemaker

f. Patients who were not eligible for this study at the discretion of the researcher

\section{Measurements}

Visual analogue scale (VAS): VAS is a commonly used method to assess treatment efficiency of musculoskeletal disease. It is also convenient and simple to use and apply for evaluation. The most severe pain or uncomfortable feeling is recorded 10 , and the state of painless is recorded $0 .{ }^{14}$

Passive range of movement (PROM): Inspector calculates participant's shoulder abduction, extension, external rotation, flexion, and internal rotation when participants complain of pain in sitting position by angulometer.

\section{Procedure}

\section{Treatment device}

Using device: electrical heating needle system SHA-21-01

Licensed number: 11-1013

Object of device: To incise and coagulate tissue

Mechanism of device: This device consists of console, monopolar needle, bipolar forceps, and bovie plate. When the current generates by console, the current flows through monopolar needle into the human body. Then, the heat is generated only in the needle tip. The monopolar needle is insulated except the needle tip, so that the heat transmission is more effectively applied only to the part of the body connected to the needle tip.

\section{Treatment methods}

A. Identify patients and confirm their eligibility

B. The participants were divided into study group $(n=10)$ and control group $(\mathrm{n}=8)$

C. The patient's baseline VAS and PROM were measured.

D. In study group, electrical fire needling treatment was conducted on pain side by a single-use $0.40 * 40 \mathrm{~mm}$ monopolar needle. The acupoints are LU2, HT1, SI9, SI11, GB21, and TE14. Treatment time is about 3 to 5 seconds.

E. In control group, the sham-electrical fire needling treatment was conducted on pain area by the same device. We set the current 0 and have the device bleep in spite of supplying 0 current, trying to have the participants feel like having the same treatments as the participants in the study groups have. The same acupoints and treatment time are applied to the control group as well as the study group.

F. The results are assessed every two and four weeks after the treatment.

\section{The expected side effects}

Although electrical fire needling device was proven efficient and safe, side effects may occur when the following ones happen.
A. Subcutaneous haemorrhage
B. Peripheral neuritis or cellulitis
C. Sore of local site
D. Spasm or burns of acupoints
E. Excessive sweating
F. Heating sense

\section{Statistical analysis}

Statistical analysis using SPSS 20.0 ver. on Windows for Statistical analysis was used to compare the variation of the numerical values. The experimental results of each figure were expressed with mean and \pm standard deviation. To compare the difference between before and after electrical fire needling treatment, Wilcoxon signed-ranks test was conducted. To compare the difference between study group and control group, Mann-Whitney U test was also conducted.

\section{Results}

\section{Demographic characteristics}

The sex, age, K-ALSFRS-R, site of onset, site of pain of the participants are given in Table 1 . These are the basic information to understand the status for ALS patient. K-ALSFRS-R: Korean-ALS Functional Rate Scale-Revised. And the baseline of VAS and PROM before treatment is given in Table 2 .

\section{The change of VAS in the course of treatment}

The pain evaluated by VAS was gradually decreased in both groups (Table 3). And there was a significant difference between study group and control group at T2 (report after 2 weeks, 4 treatment) and T4 (report after 4weeks, 8 treatment) $(\mathrm{p}<0.05)$.

In the interval difference of 2 treatment times at VAS in the study group, there was a significant difference at $\triangle 0$-2week (the changes between before treatment - 4th treatment), $\triangle 0$-4week (the changes between before treatment -8 th treatment $)(\mathrm{p}<0.05)$. In the control group, there was a significant difference at $\triangle 0$-2week, $\triangle 0$-4week $(\mathrm{p}<0.05)$ (Table 4).

And there was a significant difference between study group and control group at $\triangle 0-2$ week, $\triangle 0-4$ week $(\mathrm{p}<0.05)$ (Table 4).

The change of passive range of movement (PROM) in the course of treatment: The PROM was increased in both groups except T2 (report after 2 weeks, 4 treatments) to T4 (report after 4weeks, 8 treatments). However, there was no significant difference between study group and control group ( $\mathrm{p}>0.05)$ (Table 5).

In the interval difference of 2 Treatment times at abduction in the study group, there was a significant difference $\triangle 0$-2week (the changes during before treatment - 4th treatment), $\triangle 2-4$ weeks(the changes during 4 th treatment - 8 th treatment), $\triangle 0-4$ week(the changes during before treatment -8 th treatment $)(\mathrm{p}<0.05)$. In the interval difference of 2 treatment times at extension, external rotation, and abduction in the study group, there was a significant difference $\triangle 0-2$ week(the changes during before treatment - 4th treatment), $\triangle 0$-4week(the changes during before treatment -8 th treatment $)(p<0.05)$. At internal rotation in the study group, there was no significant difference in all periods $(\mathrm{p}>0.05)$.

In the control group, the interval difference of 2 Treatment times at abduction there was a significant difference $\triangle 0$-4week (the chan- 
ges during before treatment- 8 th treatment $)(p<0.05)$. In the interval difference of 2 treatment times at extension, there was a significant difference at $\triangle 0$-2week (the changes during before treatment- 4 th treatment), $\triangle 0-4$ week (the changes during before treatment-8th treatment) $(\mathrm{p}<0.05)$. At external rotation, there was no significant difference in all periods $(\mathrm{p}>0.05)$. At flexion, there was a significant difference in all periods $(\mathrm{p}<0.05)$. At internal rotation, there was a significant difference at $\triangle 2-4$ week (the changes during 4 th treatment-8th treatments).

When we compare study group and control group, there was no significant difference except at flexion $\triangle 0$-2week (the changes during before treatment -4 th treatment $)(p<0.05)$ (Table 6).

Table I Characteristics of patients

\begin{tabular}{|c|c|c|c|}
\hline \multirow{2}{*}{\multicolumn{2}{|c|}{ Age(mean $\pm S D$, years) }} & Study group $(n=10)$ & Control group $(n=8)$ \\
\hline & & $52.6 \pm 7.46$ & $55.13 \pm 13.34$ \\
\hline \multicolumn{2}{|c|}{ K-ALSFRS-R(mean $\pm S D)$} & $32.1 \pm 9.57$ & $28.5 \pm 12.57$ \\
\hline \multirow{2}{*}{$\operatorname{Sex}(n)$} & Male & 7 & 2 \\
\hline & Female & 3 & 6 \\
\hline \multirow{3}{*}{ Site of onset } & upper limb & I & 4 \\
\hline & lower limb & 7 & 3 \\
\hline & bulbar & 2 & 1 \\
\hline \multirow{2}{*}{ Site of pain } & right side & 6 & 6 \\
\hline & left side & 4 & 2 \\
\hline
\end{tabular}

Table 2 Baseline characteristics

\begin{tabular}{llll}
\hline & & Study group & Control group \\
\hline VAS & & $6.9 \pm 1.04$ & $7.1 \pm 1.25$ \\
PROM & Abduction & $72.9 \pm 48.73$ & $80.8 \pm 24.13$ \\
& Extension & $33.5 \pm 17.04$ & $26.6 \pm 6.02$ \\
& External rotation & $30.7 \pm 24.19$ & $38.9 \pm 6.27$ \\
& Flexion & $84.9 \pm 50.49$ & $108.9 \pm 108.88$ \\
& Internal rotation & $47 \pm 21.59$ & $43.25 \pm 5.63$ \\
\hline
\end{tabular}

Table 3 Comparison of 2 groups at the change of visual analogue scale

\begin{tabular}{llll}
\hline & T0 & T2 & T4 \\
\hline Study Group & $6.9 \pm 1.04$ & $4.7 \pm 1.57$ & $4.5 \pm 1.51$ \\
Control Group & $7.1 \pm 1.25$ & $6.5 \pm 0.93$ & $6.1 \pm 0.84$ \\
p-value & 0.736 & $0.016^{*}$ & $0.02^{*}$ \\
\hline
\end{tabular}

Values are mean $\pm \mathrm{SD}$

Analyzed by Mann Whitney $U$ test $(* p<0.05)$

TO: Primary report before $\left.\right|^{\text {st }}$ treatment

T2: Report after 2 weeks, $4^{\text {th }}$ treatment

T4: Report after 4 weeks, $8^{\text {th }}$ treatment

Table 4 Interval difference of 2 treatment periods at visual analogue scale

\begin{tabular}{|c|c|c|c|c|}
\hline & & $\triangle 0$-2week & $\triangle$ 2-4week & $\triangle 0$-4week \\
\hline \multirow{2}{*}{ Study Group } & Mean \pm SD & $2.2 \pm 1.23$ & $0.2 \pm 1.03$ & $2.4 \pm 1.26$ \\
\hline & p-value* & $0.005^{*}$ & 0.557 & $0.004 *$ \\
\hline \multirow{2}{*}{ Control Group } & Mean \pm SD & $0.6 \pm 0.74$ & $0.4 \pm 0.52$ & $I \pm 0.76$ \\
\hline & $\mathrm{p}$-value* & 0.059 & 0.083 & $0.023 *$ \\
\hline$p$-value $\dagger$ & & $0.007 \dagger$ & 0.682 & $0.012 \dagger$ \\
\hline
\end{tabular}

Values are mean $\pm S D$

*P-value:Analyzed by Wilcoxon signed-ranks test $(p<0.05)$

†p-value:Analyzed by Mann Whitney $U$ test $(p<0.05)$

$\triangle 0$-2week: The changes during before treatment-4th treatment

$\triangle$ 2-4week:The changes during 4 th treatment-8th treatment

$\triangle 0$-4week:The changes during before treatment-8th treatmen 
Table 5 Comparison of 2 groups at the change of painless passive shoulder range of movement

\begin{tabular}{lllll}
\hline & & T0 & T2 & T4 \\
\hline Abduction & Study group & $72.9 \pm 48.73$ & $87.5 \pm 48.12$ & $94.4 \pm 47.13$ \\
& Control group & $62.75 \pm 24.13$ & $68.25 \pm 19.68$ & $72 \pm 19.77$ \\
*p-value & 0.929 & 0.689 & 0.286 \\
Extension & Study group & $33.5 \pm 17.04$ & $36.8 \pm 14.47$ & $36.8 \pm 14.78$ \\
& Control group & $26.63 \pm 6.02$ & $29.63 \pm 6.44$ & $29.63 \pm 4.60$ \\
External rotation & *p-value & 0.196 & 0.305 & 0.284 \\
& Study group & $30.7 \pm 24.19$ & $37.3 \pm 24.63$ & $37.4 \pm 23.85$ \\
Flexion & Control group & $38.88 \pm 6.27$ & $40.25 \pm 7.23$ & $43.88 \pm 10.95$ \\
& *p-value & 0.422 & 0.689 & 0.475 \\
Internal rotation & Study group & $84.9 \pm 50.49$ & $109.5 \pm 41.82$ & $113.6 \pm 43.44$ \\
& Control group & $108.88 \pm 54.95$ & $111.88 \pm 54.21$ & $116.25 \pm 56.66$ \\
& *p-value & 0.373 & 0.965 & 0.214 \\
\hline
\end{tabular}

Values are mean $\pm S D$

*p-value:Analyzed by Mann-Whitney $U$ test

TO: Primary report before Ist treatment

T2: Report after 2 weeks, $4^{\text {th }}$ treatment

T4: Report after 4 weeks, $8^{\text {th }}$ treatment

Table 6 Interval Difference of 2 treatment periods at painless passive shoulder range of movement

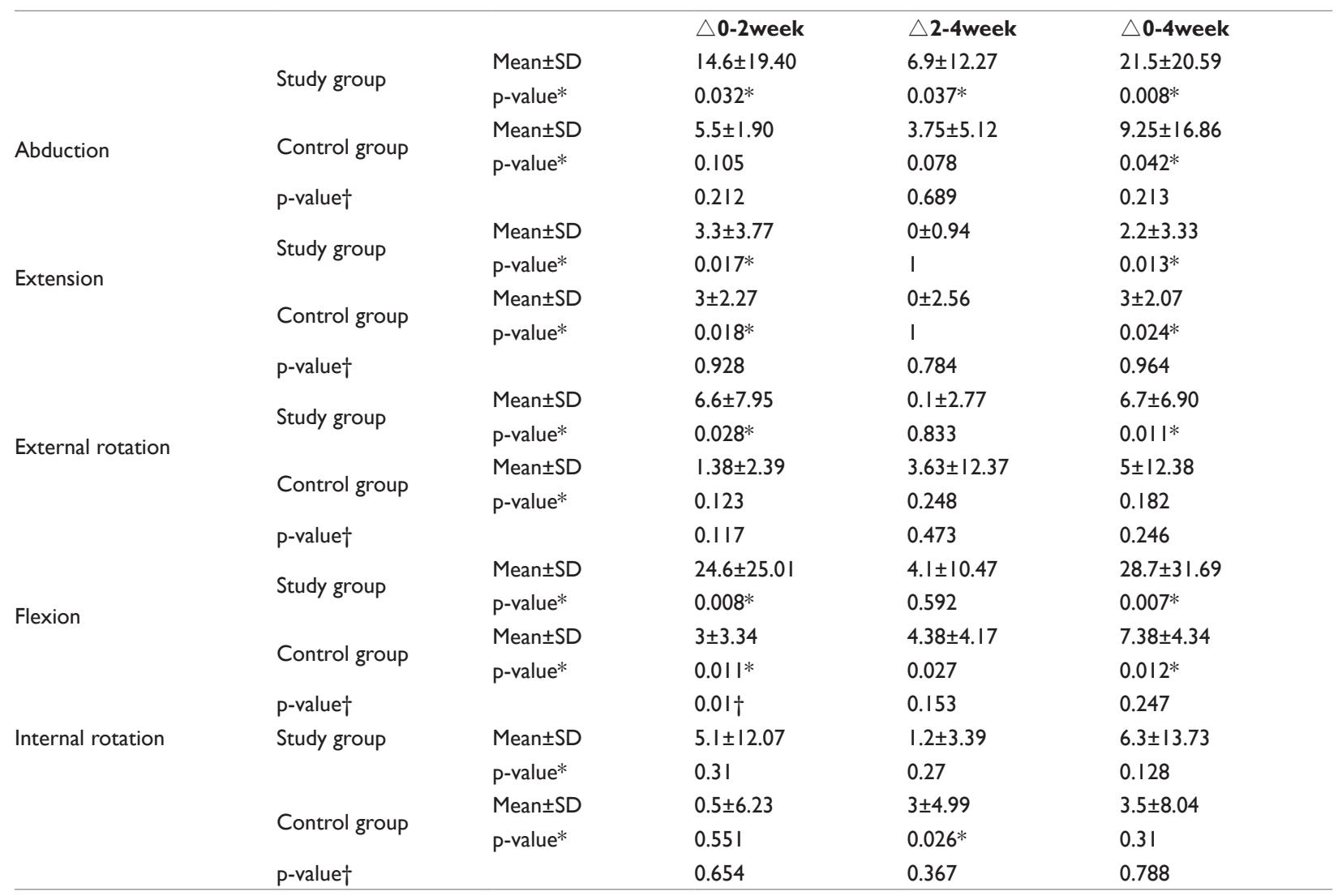

Values are mean $\pm \mathrm{SD}$

*p-value: Analyzed by Wilcoxon-signed ranks test $(p<0.05)$

tp-value:Analyzed by Mann Whitney $U$ test $(p<0.05)$

$\triangle 0$-2week: The changes during before treatment - $4^{\text {th }}$ treatment

$\triangle 2$-4week: The changes during 4th treatment - $8^{\text {th }}$ treatment

$\triangle 0$-4week:The changes during before treatment $-8^{\text {th }}$ treatment

Citation: Kim S, Cha E, Lee S, et al. Clinical effectiveness of electrical fire needling for shoulder pain of amyotrophic lateral sclerosis: a pilot study. Int J Complement Alt Med. 2016;4(2):I I-I2. DOI: 10.15406/ijcam.2016.04.00 I I 


\section{Discussion}

Amyotrophic lateral sclerosis (ALS) is a neurodegenerative disease characterized by dominant involvement of upper and lower motor neurons, leading to progressive weakness of bulbar, limb, thoracic and abdominal muscles. Within 2-4years, it causes progressive and severe muscle weakness, eventually causing death due to respiratory muscle palsy. ${ }^{15,16}$ No curative treatment is available; therefore, the mainstay of management is primarily supportive and symptomatic. ${ }^{17}$ Symptoms such as cramps, spasticity or pain should be treated first as they become prominent and incapacitating. ${ }^{16}$ Using a prospective epidemiological register, the authors found that most of the patients with ALS $57 \%$ reported pain, with $14 \%$ in pain rated as severe according to the Brief Pain Inventory. Pain in ALS was most commonly found in the extremities, shoulder, and hip. ${ }^{18}$

Shoulder pain is a very common in musculoskeletal disease. The pain or impairment of shoulder joint occurs easily because of the instability of shoulder joint. It is the third most common cause of musculoskeletal consultation in primary care. ${ }^{19,20}$ There are many causes for shoulder pain. The common causes are postural disorder, fibrositis, sprain, strain, subacromial bursitis, subdeltoid burisitis, calcific tendinitis of supraspinatus tendon, partial rupture of the rotator cuff, biceps brachii tendinitis, supraspinatus tendinitis, glenohumeral instability, operation or trauma and continuous fixation. ${ }^{21,22}$ The treatment of shoulder pain is analgesic or steroid medicine, injection of anesthetic drugs, and physical therapy ${ }^{23}$ In terms of oriental medicine, shoulder pain is all sort of pain symptoms that appear on the shoulder. The common symptoms of shoulder pain are shoulder joint pain, limitation of range of movement, hypoesthesia such as heating or chilling sense, rad pain to cervical, scapular or elbow. If the symptoms getting longer, it becomes 肩凝 and movement will be limited or even impossible. ${ }^{24}$ The causes of shoulder pain are wind, coldness, dampness, stagnation of Qi or blood, phlegm and retained fluid, bruise, traumatic accident, and seven emotion. . $^{19,24,25}$

From past to present, a lot of treatment methods have been developed to treat shoulder pain in oriental medicine11). Among them, the fire needling is a therapeutic measures of relieving pain, ${ }^{26}$ applying the stimulation of both acupuncture and heating, making meridian system become warm and pulses flow well in the body, and finally resulting in having muscles be relieved and meridian be smooth. ${ }^{27}$ We can look for the origin of the fire needling from 《・帝 - 經》 in which the name of the '燔鍼' or the '焠鍼' is called, and the acupuncture method is relevant to the ‘焠刺’. The <素門.調經論 $>$ describes like this, “病 在脈調之血, 病在脈, 調之絡, 病在氣, 調之衛, 病在肉, 調 之分肉，病在筋，病在骨，調之骨。燔鍼却刺其下及與急者病 在骨，焠銊藥慰”, which explains that the fire needling medicine is efficacious for the disease of muscle, bone, and pulse (筋，骨，脈). ${ }^{28}$ The <素門.調經論> describes that “九曰焠刺，焠者者燔鍼則取 痺也”, which explains that since the fire needling was applied to arthralgia syndrome and muscular diseases. It has been more developed with China as the center according to the passing of time to the area of muscular skeletal disease, internal medicine, gynecological medicine, and the E.N.T. ${ }^{13}$ As an extension of the fire needling, electrical fire needling device was developed lately. Having been applied to the area of many kinds of diseases such as skin disease, ${ }^{29}$ backache, ${ }^{30}$ tarsalgia, ${ }^{31}$ and haemorrhoids ${ }^{32}$ in China. Internally in Korea, several studies about the fire needling have been presented in this regard. There has been reported several studies about effectiveness of fire needling to treating biceps tendinitis, ${ }^{26}$ medial ${ }^{27}$ and lateral epicondylitis, ${ }^{33}$ trigger finger, ${ }^{34}$ and compression fracture. ${ }^{35}$
However, there has not been any study about electrical fire needling that is related and applied to shoulder pain of ALS patients. Therefore, this study was conducted to study the effect of electrical fire needling treatment for shoulder pain of amyotrophic lateral sclerosis (ALS).

The 18 eligible participants were divided into study group and control group. Prior to treatment, we checked pain VAS and shoulder passive range of movements (flexion, extension, abduction, and internal rotation) in both group. In Study group, electrical fire needling treatment was conducted on pain side for 3 to 5 seconds. The acupoints are LU2, HT1, SI9, SI11, GB21 and TE14. In control group, the sham-electrical fire needling treatment was conducted on pain area by the same device. We set the current 0 and have the device bleep in spite of supplying 0 current, trying to have the participants feel like having the same treatments as the participants in the study groups have. The same acupoints and treatment time were applied to the control group as well as the study group.

The pain evaluated by VAS was gradually decreased in both groups. Especially, the pain VAS was more significantly decreased in earlier treatment stage (before treatment to 4th treatment) than later treatment stage (4th treatment to 8th treatment) in study group. Also, there was a significant difference between study group and control group.

Being compared between before and after treatments, the results of ROM were increased, except at extension at the changes of during 4th treatment to 8th treatment in both groups. But there was no significant difference between study group and control group. In the interval difference of 2 treatment periods at range of movements, the results of ROM were more significantly increased in earlier treatment stage(before treatment to 4 th treatment) than later treatment stage(4th treatment to 8th treatment) in both group. However, there was no significant difference except at the changes during before treatment to 4th treatment $(\triangle 0-2$ week) at flexion between study group and control group.

Therefore, in this study, acupuncture and electrical fire needling treatment are both effective in pain control. However, electrical fire needling treatment is more effective than acupuncture treatment.

Furthermore, It is confirmed that the electrical fire needling treatment is more effective in alleviating subjective pain (calculated by VAS) rather than relieving stiffness of shoulder joint(calculated by ROM) in this study.

There were no side effects during this study but there were some limitations in our study. First, the extracted samples were not randomized. Second, this study was not double-blind test, so the bias could occur. Third, the sample size of this study was too small to obtain meaningful data. ALS is rare and incurable disease, so it is difficult to make a large sample size. Fourth, because the treatments were invasive in both groups, we could not rule out the placebo effect entirely. Therefore, in the future study, no treatment group or sham-acupuncture group will be needed. Fifth, this was conducted by hospitalized patients. So, scolopendrid \& bee venom pharmacopuncture was applied uniformly to all participants. Also, depending on the individual patient's symptoms, e.g., indigestion, constipation, common cold, the herbal medicine was administered a short time. ALS is incurable and progressive disease, it was not possible to ignore those symptoms for ethical reasons.

Because there have been no previous studies about the electrical fire needling treatment applied for ALS patients, this study was con- 
ducted to show a short-term effect of electrical fire needling treatment. It is necessary to complement the above-mentioned limitations and to make these results available as future clinical preliminary data. Ongoing research with the development of new acupuncture treatment and evaluation of validation should continue in the future in order to extend the life and improve the quality of life for ALS patients.

\section{Conclusion}

On the 18 ALS patients who received electrical fire needling treatment (Study group), and sham-electrical fire needling treatment (Control group) in Wonkwang University Gwangju oriental medical hospital, the figures (VAS, PROM) showed the following significant results.

1. The pain evaluated by VAS was gradually decreased. The interval difference of 2 Treatment times at VAS was a significant difference between study and control group.

2. The mobility calculated by PROM was generally increased in both study and control group. The interval differences of 2 Treatment times at PROM were no significant difference except at $\triangle 0$-2week (flexion the changes during before treatment to 4 th treatment) between study and control group.

\section{Acknowledgements}

This study was supported by the Traditional Korean Medicine R\&D Program funded by the Ministry of Health \& Welfare through the Korea Health Industry Development Institute (KHIDI) (grant number: HI11C2142).

\section{Conflicts of interest}

Author declares there are no conflicts of interest.

\section{Funding}

None.

\section{References}

1. Carlesi C, Pasquali L, Piazza S, et al. Strategies for clinical approach of neurodegeneartion in amyotrophic lateral sclerosis. Arch Ital Biol. 2011;149(1):151-167.

2. Pagnini F, Lunetta $\mathrm{C}$, Banfi $\mathrm{P}$, et al. Pain in amyotrophic lateral sclerosis: a psychological perspective. Neurol Sci. 2012;33(5):1193-1196.

3. Roh JH, Ko CN, Cho KH, et al. East-west medical study on atrophy syndrome. J Int Korean Med. 1996;17(1):93-94.

4. Kwak JM, Oh MS. The literary study on flaccidity-syndrome. Daejeon university. 2000;9(1).pp.661

5. Roelke KA, Diana JL, Weasler CM. Pain in amyotrophic latera sclerosis (ALS)-presentation, prevalence, prescriptions. Neurologogy. 2000;54:343

6. Ganzini L, Johnston WS, Hoffman WF. Correlates of suffering in ALS Neurology. 1999;52(7):1434-1440.

7. de Tommaso M, Tortelli R, Sardaro M. Features of pain in amyotrophic lateral sclerosis. Eur J Neurol. 2008;15:353-354.

8. Oliver D. The quality of care and symptom control-the effects on the terminal phase of ALS/MND. J Neurol Sci. 1996;139(Suppl):134-136.

9. Mitchell JD, Borasio GD. Amyotrophic lateral sclerosis. The Lancet. 2000;69:2031-2041.

10. Simmons Z . Management strategies for patients with amyotrophic lateral sclerosis from diagnosis through death. Neurologist. 2005;11(5):257-270.

11. The Acupuncture and Moxibustion Society. 鍼尒學 下. Seoul : 集文 黨. 2008; 41-42.

12. Choe YT. Acupuncture \& Moxibustion Medicine. Seoul: Jipmoondang. 2006; 1050 .

13. Park SJ, Ahn SG. A Literature Review and A Clinical Examination of Burning Acupuncture Therapy of using D.I.T.I. Journal of Korean Acupuncture \& Moxibustion Society. 1998;15(2):407-425.

14. The Korean pain society. Pain medicine. Seoul：君子出版社. 1997;27-29.

15. Ince PG, Lowe J, Shaw PJ. Amyotrophic lateral sclerosis: Current issues in classification, pathogenesis and molecular pathology. Neuropathol Appl Neurobiol. 1998;24(2):104-117.

16. Andersen PM, Abrahams S, Borasio GD, et al. EFNS guidelines on the clinical management of amyotrophic lateral sclerosis (MALS)revised report of an EFNS task force. European Journal of Neurology. 2012;19(3):360-375.

17. Bae JS, Hong YH, Baek W, et al. Current status of the diagnosis and management of amyotrophic lateral sclerosis in Korea: A multicenter cross-sectional study. Journal of Clinical Neurology. 2012; 8(4):293-300.

18. Chio. Reassessing received wisdom in ALS-pain is common when studied systematically. European journal of neurology. 2012;19(4):531-553.

19. Nam DW, Choi YS, Kim HB, et al. Randomized controlled trial of eastwest collaborate medical treatment on female chronic shoulder pain $\mathrm{P} \backslash$ patients. The Jounal of Korean Acupuncture\&Moxibustion Society. 2007;24(6):113-122.

20. Kim HW, Kim SS, Park SW, at al. A systematic review of randomized controlled trials on acupuncture treatment for shoulder pain. The Jounal of Korean Acupuncture\&Moxibustion Society. 2010;27(4):67-84.

21. Park JA, Lee $\mathrm{CH}$, Kwon KS, et al. Effecs of sweet bee venom pharmacopuncture on the post stroke hemiplegic shoulder pain. The Journal of Acupuncture and Moxibustion Society. 2011;28(4):37-47.

22. The Korean Orthopaedic Association, Orthopaedics(6th), Seoul 最新醫 學社. 2005;248-50, 366-77.

23. Green S, Buschbinder R, Glazier R, et al. Interventions for shoulder pain(Cochrane Review). The Cochrane Library, Oxford, England. 1999.

24. Kim KS. The clinical study on acupuncture and moxibustion for the pain of shoulder and arm. The Journal of Korean Oriental Medicine. 1984;5(1):58-61.

25. 許浚，東醫寶鑑. Seoul: 南山黨. 1976;298-299.

26. Lim GM, Kwon HY, Kim JH. A case report on the biceps tendinitis treatment with fire needling therapy. Korean Journal of acupuncture. 2010;27(3):143-150.

27. Lee KH, Ryu YJ, Sun SH, et al. The effect of burning acupuncture therapy on the traumatic injury of medial collateral ligament : Report of five cases. The Journal of Korean Acupuncture \& Moxibustion Society. 2010;27(1):140-155.

28. Shin YS, Woo SJ, Lim SI. A study of literature review on the burning acupuncture therapy. Journal of Korean Acupuncture \& Moxibustion Society. 2003; 20(4) :192-208.

29. 仲菊. 一火・治・面部粉刺104例. - 宁中医・志 1996;5:231.

30. 一学良, 黄 - , - 悦善. 一火・治・腰痛. 中国中医骨・科・志 1989;3:25.

31. - 勤, ・学良. ・ 火・治・足跟痛120例. 中国・尒 1994;1:321. 
32. 梁 - - 黄阳勇. - 火 - 治 - 症状性痔34例 - 效 - 察. 新中医 2003;35(8):55.

33. Park MK, Kang Ia, Shin MG, et al. The effect of burning acupuncture therapy on the external epicondylitis: Report of six cases. The Journal of Korean Acupuncture \& Moxibustion Medicine Society. 2012;29(4):113-121.
34. Lee CH, Park MK, Kang IA, et al. A case study of 2 trigger finger patients using burning acupuncture therapy. The Journal of Krean Acupuncture\&Moxibustion Society. 2011;28(6):169-175.

35. Lee JS, Han SH. A Case Report on Burning Acupuncture Treatments for Stable Compression Fracture. The Journal of Korean CHUNA Manual Medicine. 2002;3(1):167-174. 